\title{
Proteomic characterization of Naja mandalayensis venom
}

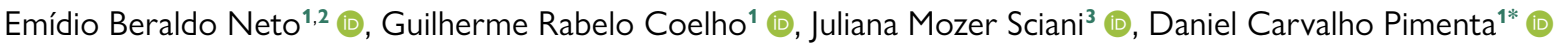 \\ ${ }^{1}$ Laboratory of Biochemistry and Biophysics, Butantan Institute, São Paulo, SP, Brazil. \\ ${ }^{2}$ Graduation Program in Science - Toxinology, Butantan Institute, São Paulo, SP, Brazil. \\ ${ }^{3}$ Multidisciplinary Research Laboratory, São Francisco University, Bragança Paulista, SP, Brazil.
}

\section{Keywords:}

Naja mandalayensis

Proteome

Spitting cobra

Three-finger toxins

SVMP

Enzymes

\begin{abstract}
Background: Naja mandalayensis is a spitting cobra from Myanmar. To the best of our knowledge, no studies on this venom composition have been conducted so far. On the other hand, few envenomation descriptions state that it elicits mainly local inflammation in the victims' eyes, the preferred target of this spiting cobra. Symptoms would typically include burning and painful sensation, conjunctivitis, edema and temporary loss of vision.

Methods: We have performed a liquid-chromatography (C18-RP-HPLC) mass spectrometry (ESI-IT-TOF/MS) based approach in order to biochemically characterize N. mandalayensis venom.

Results: A wide variety of three-finger toxins (cardiotoxins) and metallopeptidases were detected. Less abundant, but still representative, were cysteine-rich secretory proteins, L-amino-acid oxidases, phospholipases $\mathrm{A}_{2}$, venom 5 '-nucleotidase and a serine peptidase inhibitor. Other proteins were present, but were detected in a relatively small concentration.

Conclusion: The present study set the basis for a better comprehension of the envenomation from a molecular perspective and, by increasing the interest and information available for this species, allows future venom comparisons among cobras and their diverse venom proteins.
\end{abstract}

* Correspondence: dcpimenta@butantan.gov.br https://doi.org/10.1590/1678-9199-JVATITD-2020-0125

Received: 27 August 2020; Accepted: 31 March 2021; Published online: 30 July 2021 


\section{Background}

Naja mandalayensis is a spitting cobra described for the first time in 2000 by Slowinski and Wüster [1], which is endemic in the central region of Myanmar, covering the Mandalay, Magwe and Sagaing regions. Its distribution corresponds to the dry zone of Myanmar, originally an Acacia savanna that is currently an agricultural region. However, this species has adapted and thrived in the agricultural fields and village surroundings. Naja mandalayensis belongs to a parafiletic group that comprises $N$. mossambica, N. annulifera and it is closer to N. siamensis, $N$. kaouthia and N. atra. [1].

The first description of a N. mandalayensis envenoming was published by a Myanmar research group that reported eight patients that were spat in the eyes by this venomous snake. Patients reported a burning and painful sensation (ophthalmia) and presented conjunctivitis, edema and temporary loss of vision $[2,3]$. This is very similar to the N. mossambica accident, whose venom, when in contact with eyes, induces lacrimation, blepharospasm, conjunctivitis, keratitis and iritis miosis or mydriasis, symptoms related to inflammatory and cytotoxic compounds [4]. Slowinski, in the year 2000, published a letter depicting a self-accident with a $N$. mandalayensis specimen that spat venom directly to the author's eyes inducing a burning painful reaction, and conjunctivitis, according to the report [1].

Despite the lack of reports on $N$. mandalayensis accidents, they remain a serious public health issue in Myanmar, as the snakebite incidence is high, and $70 \%$ of the population live in rural areas. Treatment for these patients is mainly based on antivenom administration (although traditional methods, such as herbal medicines, electric shock and suction are still being used). Regardless of the availability of antivenoms, Myanmar faces difficulties with the supply of antivenom, particularly in the rural areas [5]. Recently, it has been reported that antiserum used in the region of Myanmar displays lower efficacy against N. mandalayensis [3].

Considering that snakebite is a serious medical problem in rural areas of Myanmar and the data available on N. mandalayensis venom and envenomation is still scarce, and taking into account the relevance of understanding the biochemical composition of its venom, we have biochemically described, for the first time, the protein composition of its venom by using high performance liquid chromatography and mass spectrometry ESI-IT-TOF (proteomic) techniques.

\section{Methods}

\section{Reagents}

All reagents used in the present study are of analytical grade and obtained from Merck (Merck KGaA, Darmstadt, Germany and/or its affiliates), unless otherwise stated.

\section{Venom attainment}

A stablished partnership between Butantan Institute and the Ministry of Health of Myanmar, encompassed in a project called 'Methods and techniques improvement for antivenom production in Myanmar' granted us access to the pooled venom sample - from captivity individuals.

\section{Sample preparation}

The first step was to decomplex the venom in order to improve mass spectrometric analyses. Briefly, $10 \mathrm{mg}$ lyophilized $N$. mandalayenses venom were resuspended in $0.1 \%$ Trifluoroacetic Acid (TFA) and centrifuged $(10,000 \times \mathrm{g})$ for 10 minutes, at $4^{\circ} \mathrm{C}$. The supernatant was then analyzed and fractionated by ReversedPhase High Performance Liquid Chromatography (RP-HPLC) in a Shimadzu Prominence binary system (Shimadzu, Kyoto, Japan), coupled to a C18 analytical column (Supelco, $250 \times 4.6 \mathrm{~mm}, 10$ $\mu \mathrm{m}$ ). UV detection was performed (SPDM 20A, Shimadzu, $\lambda$ $=214 \mathrm{~nm}$ ) and separation was achieved by a linear gradient of $0-40 \%$ solvent B (90\% acetonitrile, containing $0.1 \%$ TFA) over A $\left(0.1 \%\right.$ TFA) for 40 minutes at a constant flow of $1 \mathrm{~mL} \cdot \mathrm{min}^{-1}$.

\section{Mass spectrometry analyses}

Manually collected fractions (50 $\mu \mathrm{L}$ aliquots) were submitted to in-solution digestion under the following conditions: (1) 5 $\mu \mathrm{L}$ DTT (100 mM dithiothreitol) were added for 30 minutes at $60^{\circ} \mathrm{C}$; (2) $2.5 \mu \mathrm{L}$ of iodoacetamide $(200 \mathrm{mM})$ added for another 30 minutes at room temperature and protected from light; (3) sample incubation for at least 12 hours at room temperature with $10 \mu \mathrm{L}$ of trypsin ( $40 \mathrm{ng} / \mu \mathrm{L}$ in $100 \mathrm{mM}$ ammonium bicarbonate). The reaction was stopped by adding 50\% ACN/5\% TFA.

The samples then were analyzed by liquid chromatographymass spectrometry in an ESI-IT-TOF instrument coupled to a UPLC 20A Prominence (Shimadzu, Kyoto, Japan). Samples (15 $\mu \mathrm{L}$ aliquots) were loaded into a C18 column (Kinetex C18, $5 \mu \mathrm{m}$; $50 \times 2.1 \mathrm{~mm}$ ) and fractionated by a binary gradient employing as solvents (A) water: DMSO: acid (949: 50: 1) and (B) ACN: DMSO: water: acid (850: 50: 99: 1). An elution gradient of 0-40\% $\mathrm{B}$ was applied for 35 minutes at a constant flow of $0.2 \mathrm{~mL} . \mathrm{min}^{-1}$ after initial isocratic elution for 5 minutes. The eluates were monitored by a Shimadzu SPD-M20A PDA detector before being injected into the mass spectrometer.

The interface was kept at $4.5 \mathrm{kV}$ and $275^{\circ} \mathrm{C}$. Detector operated at $1.95 \mathrm{kV}$ and the argon collision induced fragmentation was set at 55 'energy' value. MS spectra were acquired in positive mode, in the $350-1400 \mathrm{~m} / \mathrm{z}$ range and MS/MS spectra were collected in the 50 to $1950 \mathrm{~m} / \mathrm{z}$ range.

\section{Proteomic data processing}

Raw LCD LCMSolution Shimadzu data were converted into MGF by the LCMSolution (PRIDE) tool and then loaded into 
Peaks Studio V7.0 (BSI, Canada). Data were processed according to the following parameters: MS and MS/MS error mass were $0.1 \mathrm{Da}$; methionine oxidation and carbamidomethylation as variable and fixed modification, respectively; trypsin as cleaving enzyme; maximum missed cleavages (3), maximum variable PTMs per peptide (3) and non-specific cleavage (both); the false discovery rate was adjusted to $\leq 0.5 \%$; only proteins with score $\geq 20$ and containing at least 1 unique peptide were considered in this study. Data were analyzed against a Naja protein database (963 entries) compiled on January 2020 and built by retrieving all UniProt entries associated with this taxon, although broad searches against the whole UniProt were performed, as well, as quality controls (data not shown).

\section{Results and discussion}

Sample preparation is a critical step, required prior to any analytical step, particularly when the relative concentration range of the sample components is wide. One possible approach is to decomplex the sample by RP-HPLC [6]. In this work, we have chosen this approach in order to make it possible to identify both major toxins and minor components. Figure 1 presents the annotated RP-HPLC chromatogram, in which the proteomically identified proteins are indicated above the correspondent chromatographic fraction. It is possible to observe that the dynamic concentration range of toxins, in this particular venom, is very wide. For example, RT $35^{\prime}$ contains six abundant proteins, which detection saturated the UV signal, whereas RT $\sim 5-25$ contains sixteen minor proteins, which UV detection level was, at least, 15 times less intense than the largest signal. Thus, if shotgun proteomic strategy would be performed, less intense proteins would not likely be detected, as presented here. Although other sample preparation methods are available, such as batch-ion exchange chromatography [7], we have chosen to perform RP-HPLC based separation for our previous studies have demonstrated the non-viscous venoms can be efficiently fractionated by this technique $[8,9]$.

Therefore, this decomplexation step - which was not intended to yield an 'analytical' profile, with the best possible resolution and signal to noise ratio - made it possible to normalize the protein contents in each individual fraction, leading to an optimal sample processing (reduction, alkylation and trypsinization) and consequent enhanced mass spectrometric detection of the normalized tryptic peptides.

Following sample preparation and proteomic processing, $N$. mandalayeneses venom revealed itself to be a complex mixture of proteins (Figure 2). The UniProt GO molecular function annotation analyses led to the protein distribution presented in Figure 2A. Not surprising, more than half of the identified proteins fell into the 'toxin' category. In order to better understand these toxins, a second pie-chart graph was assembled analyzing only the 'toxin' keyword matched proteins (Figure 2B). This toxin distribution is in agreement with the recent work of Kazandjian et al. [10] that have elegantly studied the convergent evolution of the venom components of spitting cobras.
Among the identified proteins, some known venom toxins could be detected. Namely: venom zinc metalloproteinase (SVMP) (Table 1), phospholipase $\mathrm{A}_{2}\left(\mathrm{PL} \mathrm{A}_{2}\right)$, L-amino acid oxidase (LAAO), cysteine-rich venom protein (CRISP), venom 5-nucleotidase (V5N) and venom nerve growth factor (VNGF) (Table 2) [11, 12]. Moreover, other proteins, such as venom phosphodiesterase and NADH-related enzymes could be identified, also shown in Table 2. The complete list of the 'other' identified proteins is presented in the Additional file 1.

The pharmacological effect of the SVMPs in cobras has not been fully understood yet [13]. Few cobra SVMPs were biologically studied. Kaouthiagin (uniprot entry P82942) is an example: it seems to specifically bind to and cleave von Willebrand factor. In this sense, those authors speculate that this enzyme could be used as a pharmacological tool for functional studies of this factor [14].

Mocarhagin (Q10749) is another Naja SVMP that alters the clotting homeostasis [15]. But, in general, most cobra's SVMPs have only been associated with platelet aggregation inhibition [16]. In the present work we have observed that - for N. mandalayensis - SVMPs were the most abundant (Figure 1) proteins identified among the hydrolases. The proteomic analyses (Table 1) show that these enzymes matched SVMPs from three different Naja species. It is interesting to mention that the high peptide count presented in Table 1 not only indicates that several peptides were detected in the MS analyses (protein quantity), but also that these peptides are fairly distributed throughout the protein (rather than matching only the active site, for example), indicating that there is some degree of protein homology among these species (Figure 3) [17, 18, 19].

LAAO and PLA $\mathrm{A}_{2}$ are commonly highlighted as enzymes of medical importance $[20,21]$ due to their abundance in the venom. These proteins are presented in Table 2, among others. The currently idenfied Naja LAAO (A8QL58) has little reported information regarding its pharmacological effects. Thus, this protein could present itself as an interesting molecule for further studies, for it is a remnant isoform from a diverse family of toxins. The here reported $\mathrm{PLA}_{2}$ (P14556), is similar to Nigexine - a basic phospholipase $\mathrm{A}_{2}$ from the venom of the spitting cobra Naja nigricollis - and may display an anticoagulant activity and affect the neuromuscular transmission [21, 22]. PLA 2 s from $N$. naja are considered lethal due to their neurotoxic activity, as previously observed in an experimental envenomation study [23]. Moreover, LAAOs, $\mathrm{PLA}_{2} \mathrm{~s}$ and SVMPs have been associated to tissue inflammation and local necrosis of Elapidae snakes [24].

Also presented in Table 2 is the identification of Venom Phosphodiesterase (A0A2D0TC04) and V5N (A0A2I4HXH5), proteins that affect homeostasis and inhibit platelet aggregation induced by ADP, a consequence of both enzymes being able to degrade nucleotides [25-29]. According to Mitra and Bhattacharyya [30] who have isolated a phosphodiesterase from Daboia russelli venom with ADP hydrolytic activity, the platelet aggregation inhibition (in a human platelet rich plasma model) would be a consequence of the enzyme's activity. 


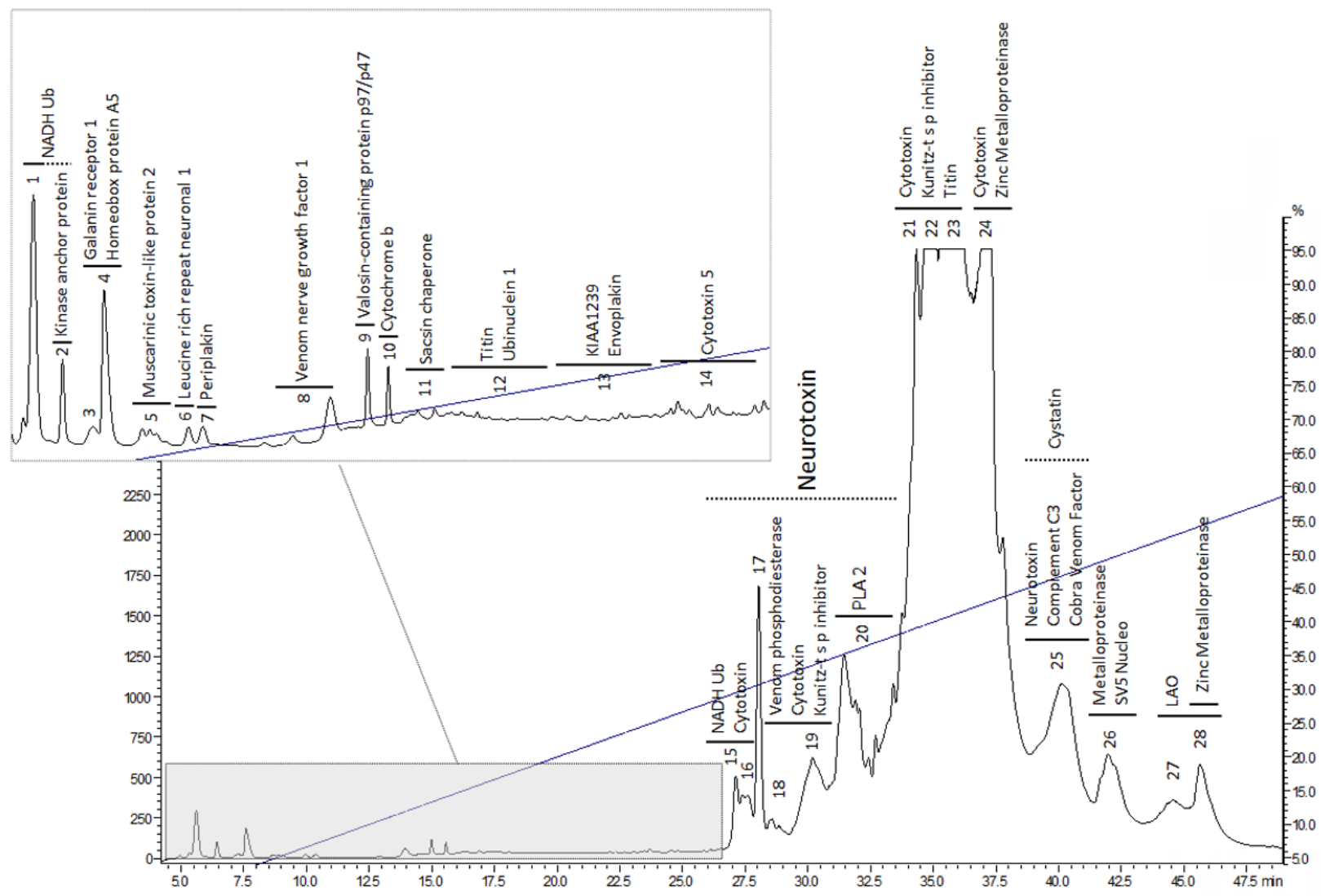

Figure 1. C18-RP-HPLC representative profile of N. mandalayensis venom solution containing the fraction numbering as well as the toxin identification attained after the fraction proteomic processing.

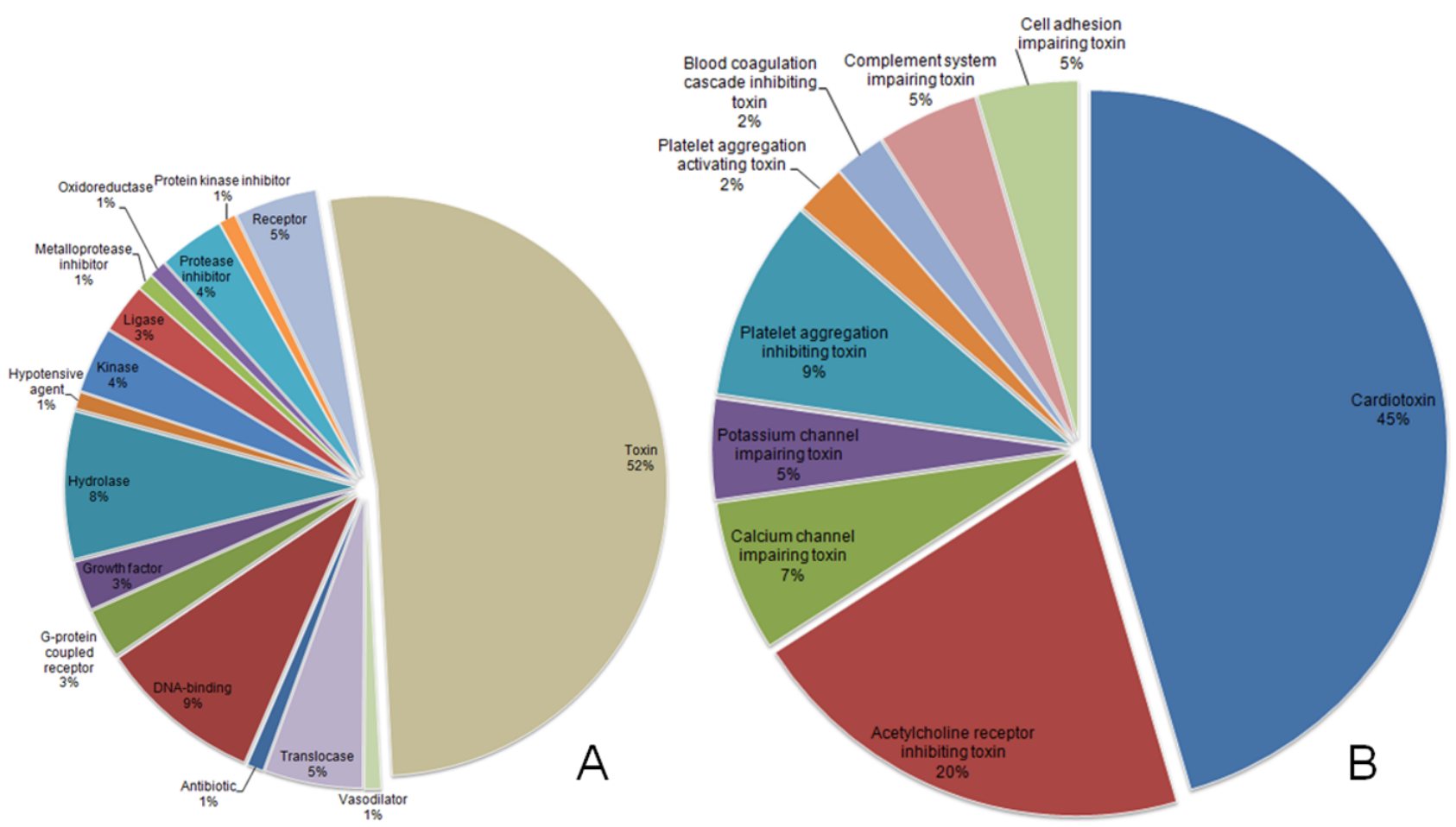

Figure 2. (A) Relative protein distribution (according to their UniProt 'molecular function' identifier) of the proteomically identified proteins in N. mandalayensis venom. (B) Relative toxin (from 'A') distribution (according to their UniProt 'mechanism of action' identifier) of this proteomic subset. 
Table 1. Known metallopeptidases matched to the proteomically identified toxins from N. mandalayensis venom.

\begin{tabular}{|c|c|c|c|c|c|}
\hline Description & Accession & $-10 \lg P^{1}$ & Peptides $^{2}$ & Avg. mass $^{3}$ (Da) & Organism $^{4}$ \\
\hline Zinc metallopeptidase-disintegrin-like atragin & D3TTC2 & 319.7 & 25 & 69181 & N. atra \\
\hline Zinc metallopeptidase-disintegrin-like cobrin & Q9PVK7 & 259.8 & 23 & 67662 & \\
\hline $\begin{array}{l}\text { Snake venom metallopeptidase-disintegrin-like } \\
\text { mocarhagin }\end{array}$ & Q10749 & 249.9 & 20 & 68176 & N. mossambica \\
\hline $\begin{array}{l}\text { Zinc metallopeptidase-disintegrin-like } \\
\text { atrase-A }\end{array}$ & D5LMJ3 & 214.4 & 23 & 68254 & N. atra \\
\hline
\end{tabular}

${ }^{1}$ Peaks Suite confidence parameter. The cutoff was set $>50 .{ }^{2}$ Matched peptides supporting the protein identification. ${ }^{3}$ Retrieved theoretical value. ${ }^{4}$ Species from which the matching toxins were identified.

Table 2. Other known enzymes matched to the proteomically identified toxins from N. mandalayensis venom.

\begin{tabular}{|c|c|c|c|c|c|}
\hline Description & Accession & $-10 \lg P^{1}$ & Peptides $^{2}$ & Avg. mass $^{3}$ (Da) & Organism $^{4}$ \\
\hline L-amino-acid oxidase & A8QL58 & 323.01 & 43 & 57963 & \multirow{3}{*}{ N. atra } \\
\hline Snake venom 5'-nucleotidase (Fragment) & $\mathrm{A} 0 \mathrm{~A} 214 \mathrm{HXH} 5$ & 216.74 & 25 & 58198 & \\
\hline Venom phosphodiesterase & A0A2D0TC04 & 186.34 & 22 & 94616 & \\
\hline Basic phospholipase $\mathrm{A}_{2}$ nigexine & P14556 & 56.76 & 2 & 13340 & N. pallida \\
\hline A kinase anchor protein 9 & $\mathrm{~K} 4 \mathrm{G} \times 13$ & 47.94 & 4 & 47420 & N. kaouthia \\
\hline \multirow{6}{*}{$\begin{array}{l}\text { NADH-ubiquinone } \\
\text { oxidoreductase chain } 2\end{array}$} & A9X4E0 & & 2 & 38341 & N. naja \\
\hline & Q2V505 & & 2 & 38341 & N. atra \\
\hline & A0A4P2VGL4 & 42.95 & 2 & 37925 & N. kaouthia \\
\hline & B6DCF5 & & 2 & 38321 & \multirow{2}{*}{ N. atra } \\
\hline & B6DA70 & & 2 & 38253 & \\
\hline & Q8W9X2 & 37.31 & 2 & 38059 & N. nivea \\
\hline $\begin{array}{l}\text { NADH-ubiquinone } \\
\text { oxidoreductase chain } 4\end{array}$ & D9YM78 & 35.69 & 2 & 23869 & N. arabica \\
\hline \multirow{3}{*}{$\begin{array}{l}\text { Phosphatidylinositol-4,5-bisphosphate } \\
\text { 3-kinase catalytic subunit gamma }\end{array}$} & A0A5B9CKM0 & \multirow{3}{*}{30.63} & 2 & 37581 & N. atra \\
\hline & A0A5B9CL15 & & 2 & 37581 & N. kaouthia \\
\hline & A0A5B9CMR9 & & 2 & 37597 & \multirow[t]{3}{*}{ N. atra } \\
\hline \multirow{6}{*}{$\begin{array}{l}\text { NADH-ubiquinone } \\
\text { oxidoreductase chain } 4\end{array}$} & A0A1W5PVT2 & & 1 & 24190 & \\
\hline & A0A3G2KUZ9 & & 1 & 24434 & \\
\hline & A0A3G2KV06 & & 1 & 24390 & \multirow[t]{3}{*}{ N. melanoleuca } \\
\hline & A0A3G2KV13 & & 1 & 24450 & \\
\hline & A0A3G2KV16 & 26.51 & 1 & 24420 & \\
\hline & A0A3G2KV47 & & 1 & 24445 & N. peroescobari \\
\hline \multirow{3}{*}{ NADH dehydrogenase subunit 4} & A0A3G2KUY9 & & 1 & 24469 & \multirow{3}{*}{ N. guineensis } \\
\hline & A0A3G2KV04 & & 1 & 24481 & \\
\hline & A0A3G2KV05 & & 1 & 24481 & \\
\hline
\end{tabular}

${ }^{1}$ Peaks Suite confidence parameter. The cutoff was set $>25$. ${ }^{2}$ Matched peptides supporting the protein identification. ${ }^{3}$ Retrieved theoretical value. ${ }^{4}$ Species from which the matching toxins were identified. 


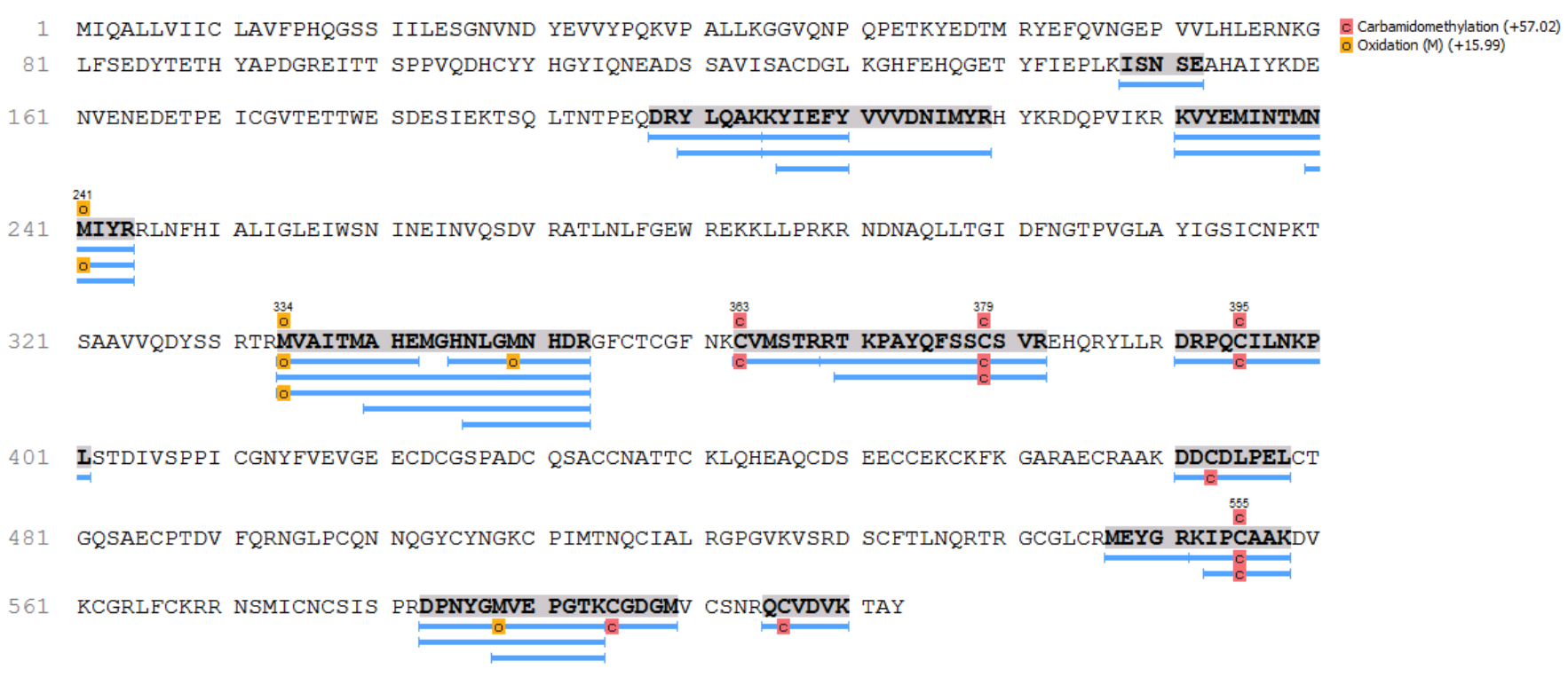

Figure 3. Coverage map representing the proteomic identification of SVMP (D3TTC2), according to Peaks Studio analyses. Blue bars represent the proteomically matched peptides, from N. mandalayensis venom, over the deposited sequence from N. atra. Letters ' $c$ ' and 'o' are the considered post-translation modifications selected for this analysis.

Some toxins, on the other hand, do not display a defined role in the venom yet, such as Phosphatidylinositol-4,5-bisphosphate 3-kinase (A0A5B9CKM0), the NADH metabolism-related enzymes (Table 2) and A-kinase anchor protein 9 (K4GX13). Although the latter is directly related to the control of the production of reactive oxygen species in cardiac stress, responsible for cardiomyocyte dysfunction and death [31].

Nonetheless, the majority of the identified proteins in $N$. mandalayensis venom were three-finger toxins (3FTx, Figure 2B), particularly cytotoxins (Table 3) and neurotoxins (Table 4). Such abundance of 3FTx has been reported by other authors for related species [10]. In a work that describes the proteome of $N$. kaouthia and $N$. naja venom, $3 \mathrm{FTx}$ were the most representative toxins found in an Elapidae database [32].

In the present report, we could identify 19 different cytotoxins (Table 3) corresponding to approximately $45 \%$ (Figure $2 \mathrm{~B}$ ) of the toxins found. These molecules are also the ones displaying the largest presence of isoforms [33]. Their biological effects include systolic cardiac arrest and severe tissue necrosis, amongst others [34]. Moreover, most of these toxins are cytolytic to different cell lines, through a pore-forming activity on membranes. Nevertheless, their actual mechanism of action has not yet been completely elucidated [35].

The 3FTx neurotoxins (12 identifications, Table 4), accounted for about $32 \%$ of the toxins in the N. mandalayensis venom (Figure 2B). Elapid snake toxins are generally known to act on the nervous system, inhibiting the synaptic transmission by specifically and potently blocking a variety of ion channels, like $\mathrm{Ca}^{2+}$ and $\mathrm{K}^{+}$, as well as the nicotinic acetylcholine receptors [36, 37]. There are described CRISP toxins that also can behave as neurotoxins [38] by inhibiting $\mathrm{Ca}^{2+}$-activated $\mathrm{K}^{+}$channels and voltage-gated $\mathrm{K}^{+}$channels. Table 3 lists the $3 \mathrm{FTx}$-neurotoxins found in the current work.
In order to compare the present identified proteins groups/ families and glimpse on their biological interest/relevance, we have compared the relative venom protein composition of $N$. mandalayensis venom with $N$. kaouthia, the closest phylogenetically relative according to Slowinski and Wüster [1]. This analysis is presented in Figure 4 and shows that homologous proteins display similar relative concentrations, such as acetylcholine receptor inhibitors, calcium channel inhibitors, platelet aggregation inhibitors and complement system inhibitors.

There were, however, N. kaouthia proteins that did not match any protein in the $N$. mandalayensis venom, such as ryanodinesensitive calcium-release channel impairing and G-protein coupled acetylcholine receptor impairing. We cannot, though, relate the absence of these proteins - in the current study - to any actual lack of these proteins in N. mandalayensis venom. This event might be related to methodological/artefactual/ biological phenomena that still need to be further explored.

Noteworthy to mention is the high relative concentration of the cardiotoxins (3FTx) standing out for N. mandalayensis venom in comparison to N. kaouthia. A similar protein profile, however, has already been described for Ophiophagus hannah [25], a Malaysian king cobra. The transcriptome and proteome analyses revealed that $3 \mathrm{FTx}$ were the major venom component. O. hannah's venom induces neurotoxicity and paralysis of the respiratory muscles and frequently provokes an extensive tissue necrosis and inflammation [25], similar to N. mandalayensis venom [2,3].

Elapidae and Viperidae venoms have already been described to contain multiple peptidase inhibitors, most of them belonging to the Kunitz-type pancreatic trypsin inhibitors family [39,32]. Elapidae venoms, in particular, display strong inhibitory activity over mammalian serine peptidases, including trypsin, a-chymotrypsin, plasmin and kallikrein [40]. 
Table 3. Known 3FTx cytotoxins matched to the proteomically identified toxins from $N$. mandalayensis venom.

\begin{tabular}{|c|c|c|c|c|c|}
\hline Description & Accession & $-10 \lg P^{1}$ & Peptides $^{2}$ & Avg. mass ${ }^{3}$ (Da) & Organism $^{4}$ \\
\hline Cytotoxin 1 & P01455 & 74.98 & 3 & 6696 & N. annulifera \\
\hline \multirow[t]{2}{*}{ Cytotoxin $1 \mathrm{~d} / 1 \mathrm{e}$} & Q98958 & 129.4 & 9 & 8992 & \multirow{2}{*}{ N. atra } \\
\hline & P01442 & 82.64 & 4 & 9041 & \\
\hline \multirow[t]{2}{*}{ Cytotoxin 2} & P01445 & 150.2 & 5 & 6745 & N. kaouthia \\
\hline & P01474 & 74.49 & 3 & 6850 & N. melanoleuca \\
\hline Cytotoxin 3 & P01446 & 150.2 & 5 & 6717 & N. kaouthia \\
\hline \multirow{2}{*}{ Cytotoxin 4} & P01452 & 90.91 & 3 & 6715 & \multirow[t]{2}{*}{ N. mossambicc } \\
\hline & P01443 & 82.64 & 4 & 9084 & \\
\hline \multirow[t]{2}{*}{ Cytotoxin 4N } & Q9W6W9 & 103.9 & 5 & 9099 & \multirow{2}{*}{ N. atra } \\
\hline & P07525 & 139.9 & 4 & 6810 & \\
\hline \multirow[t]{2}{*}{ Cytotoxin 5} & Q98961 & 128.6 & 4 & 9086 & \\
\hline & P24779 & 132.5 & 6 & 6654 & N. kaouthia \\
\hline Cytotoxin 5a & O73857 & \multirow{2}{*}{82.64} & 4 & 9041 & \multirow{2}{*}{ N. sputatrix } \\
\hline Cytotoxin 5b & P60310 & & 4 & 9055 & \\
\hline Cytotoxin 6 & P80245 & 136.3 & 8 & 8980 & N. atra \\
\hline \multirow{2}{*}{ Cytotoxin 7} & P86382 & 118.6 & 6 & 6792 & N. naja \\
\hline & P49122 & 54.72 & 4 & 9086 & \multirow{2}{*}{ N. atra } \\
\hline \multirow{2}{*}{ Cytotoxin 8} & Q91124 & 114.4 & 6 & 8900 & \\
\hline & P86540 & 98 & 5 & 6793 & \multirow{2}{*}{ N. naja } \\
\hline Cytotoxin 10 & P86541 & 78.19 & 3 & 6764 & \\
\hline \multirow{2}{*}{ Cytotoxin 11} & P62394 & \multirow{2}{*}{50.31} & 2 & 6842 & N. haje haje \\
\hline & P62390 & & 2 & 6842 & N. annulifera \\
\hline Cytotoxin 13 & A0A0U4N5W4 & 150.2 & 5 & 7947 & N. naja \\
\hline Cytotoxin SP15d & P60309 & 152.2 & 9 & 6652 & N. atra \\
\hline Cytotoxin 16 & A0A0U4W6K7 & 132.1 & 7 & 7967 & N. naja \\
\hline \multirow[t]{2}{*}{ Cytotoxin homolog } & P14541 & 83.26 & 2 & 6994 & \multirow[t]{2}{*}{ N. kaouthia } \\
\hline & E2ITZ4 & 124.5 & 4 & 9139 & \\
\hline \multirow[t]{2}{*}{ Three-finger toxin } & E2ITZ6 & 90.36 & 5 & 9858 & \multirow[t]{2}{*}{ N. atra } \\
\hline & E2ITZ7 & 76.54 & 3 & 8834 & \\
\hline
\end{tabular}

${ }^{1}$ Peaks Suite confidence parameter. The cutoff was set $>50 .{ }^{2}$ Matched peptides supporting the protein identification. ${ }^{3}$ Retrieved theoretical value. ${ }^{4}$ Species from which the matching toxins were identified.

Table 4. Known neurotoxins from 3FTx and CRISP families matched to the proteomically identified toxins from N. mandalayensis venom.

\begin{tabular}{|c|c|c|c|c|c|}
\hline Description & Accession & $-10 \lg P^{1}$ & Peptides $^{2}$ & Avg. mass ${ }^{3}$ (Da) & Organism $^{4}$ \\
\hline Cysteine-rich venom protein kaouthin-1 & P84805 & \multirow{2}{*}{185.4} & 9 & 26846 & N. kaouthia \\
\hline Cysteine-rich venom protein natrin-1 & Q7T1K6 & & 9 & 26882 & \multirow{2}{*}{ N. atra } \\
\hline Cobrotoxin-b & P80958 & 124.5 & 4 & 9139 & \\
\hline Cobrotoxin-c & P59276 & \multirow{3}{*}{136.8} & 4 & 6859 & N. kaouthia \\
\hline \multirow{2}{*}{ Short neurotoxin 1} & P60774 & & 4 & 6818 & N. samarensis \\
\hline & P60773 & & 4 & 6873 & N. philippinensis \\
\hline Neurotoxin 5 & P60772 & & 4 & 6818 & N. sputatrix \\
\hline Weak neurotoxin NNAM2 & Q9YGI4 & \multirow{5}{*}{59.92} & 3 & 9899 & N. atra \\
\hline Weak neurotoxin 5 & O42255 & & 3 & 9806 & \multirow{4}{*}{ N. sputatrix } \\
\hline Weak neurotoxin 6 & O42256 & & 3 & 9807 & \\
\hline Weak neurotoxin 8 & Q802B3 & & 3 & 9809 & \\
\hline Weak neurotoxin 9 & Q9W7I3 & & 3 & 9836 & \\
\hline Muscarinic toxin-like protein 2 & P82463 & 46.55 & 3 & 7298 & N. kaouthia \\
\hline
\end{tabular}

${ }^{1}$ Peaks Suite confidence parameter. The cutoff was set $>40 .{ }^{2}$ Matched peptides supporting the protein identification. ${ }^{3}$ Retrieved theoretical value. ${ }^{4}$ Species from which the matching toxins were identified. 


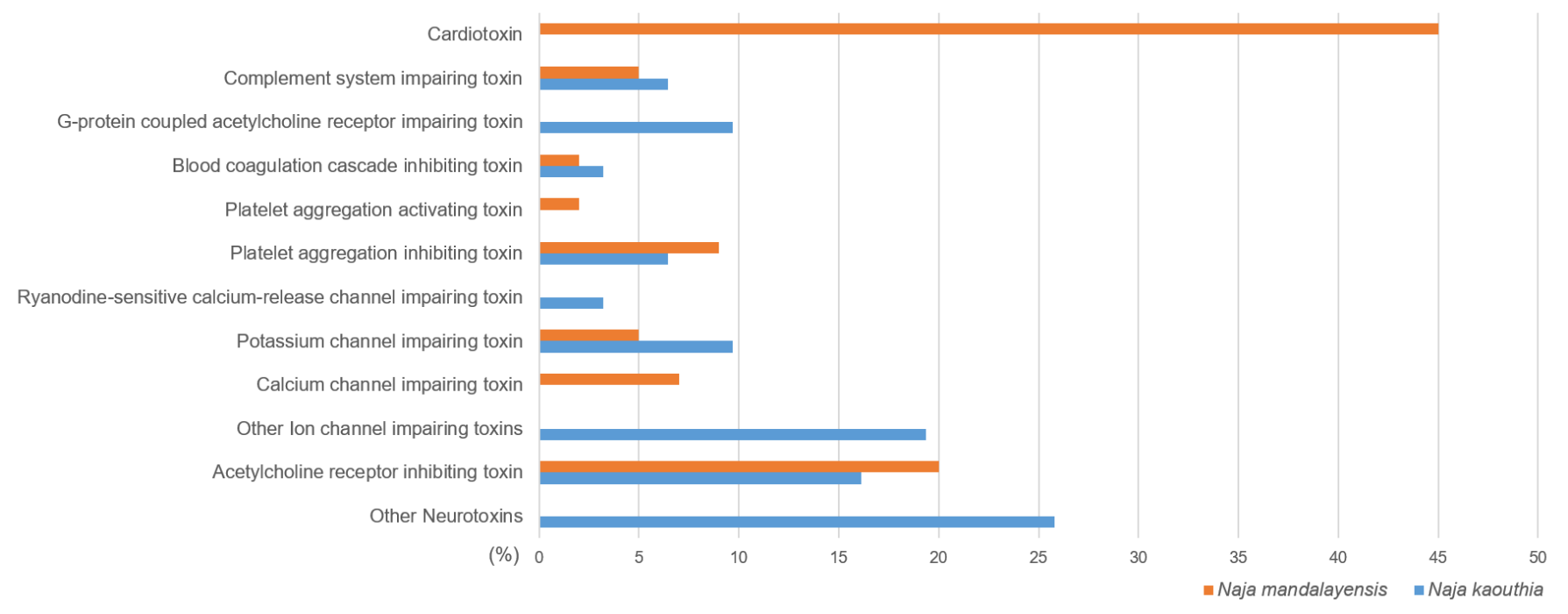

Figure 4. Relative toxin concentration distribution (\%) for N. mandalayensis (current work) and N. kaouthia (UniProt data) venoms. Classification terms based on the UniProt 'mechanism of action'.

As presented in Figure 2A, peptidase inhibitors were also detected in the studied venom; however, at low levels $(\sim 1 \%)$. Nevertheless, due to the possible physo(patho)logical effects of peptidase inhibitors, we chose to present our findings regarding these molecules in Table 5. Two Kunitz-type serine protease inhibitor were detected and, according to Peaks Studio analyses, are identical (100\% coverage, data not shown) to already sequence inhibitors from other Najas (P20229; P00986). Moreover, one cystatin already described for N. atra, (P81714), capable of inhibiting various cysteine proteases including cathepsin L, S, $B$ and papain, were, also present in the venom [39]. However, in previous studies no toxic effects have been directly associated to these molecules [32].

\section{Conclusion}

The current work comprises the first characterization of the venom proteome of $N$. mandalayenses. The findings presented here will enhance future Naja venom comparative studies, as 86 $N$. mandalayensis proteins can now be encompassed in future research from proteomic and/or evolutionary perspectives. The selected approach, i.e., the initial venom fractionation by RPHPLC, allowed mass spectrometric analyses optimization by removing the large concentration of the dynamic range variation naturally present in the venom, thus allowing the characterization of different toxins with high reliability. There are three major findings in the present study: (1) 3FTx are the major components of the venom; (2) SVMPs are the most diverse group of enzymes found, and; (3) the molecular diversity of the venom is likely to be a direct consequence of the venom spitting evolutionary strategy developed by N. mandalayensis, when compared other Naja venoms. Regarding the later observation, one can speculate that the most efficiently absorbed molecules (mucosa) would have been selected (as toxins) throughout evolution, such as the cardiotoxins here reported (Figure 2B). The current proteome description should help shed a light on the evolutionary and phylogenetic relations between $N$. mandalayenses and other Naja, since the current species, although geographically limited to southeastern Asia and consequently related to the Asian nonspitting cobras, has adopted the spitting strategy present either on the insular southeastern countries or in the African elapids.

\section{Acknowledgements}

We are thankful to Dr. Win Aung, from the Department of Medical Research of Myanmar, who kindly provided the venom samples.

\section{Availability of data and materials}

The datasets generated during the current study are available from the corresponding author on reasonable request.

\section{Funding}

This work was supported by the Coordination for the Improvement of Higher Education Personnel (CAPES - grant $\# 001$ to EBN), Financier of Studies and Projects (FINEP - grants $\#$ 01.09.0278.04 and \#01.12.0450.03), the National Council for Scientific and Technological Development (CNPq-406385/20181 to DCP) and São Paulo Research Foundation (FAPESP - grant $\# 15 / 13142-3$ to GRC). DCP is a CNPq fellow researcher (grant \#301974/2019-5).

\section{Competing interests}

The authors declare that they have no competing interests. 
Table 5. Known peptidase inhibitors matched to the proteomically identified toxins from $N$. mandalayensis venom.

\begin{tabular}{lccccc}
\hline Description & Accession & 10lgP $^{1}$ & Peptides $^{\mathbf{2}}$ & Avg. mass $^{\mathbf{3}}$ (Da) & Organism $^{\mathbf{4}}$ \\
\hline Kunitz-type serine protease inhibitor & P20229 & 187 & 7 & 6371 & N. naja \\
Kunitz-type serine protease inhibitor 2 & P00986 & 117 & 3 & 6466 & N. nivea \\
Cystatin & P81714 & 20.33 & 1 & 10995 & N. atra \\
\hline
\end{tabular}

${ }^{1}$ Peaks Suite confidence parameter. The cutoff was set $>50$. ${ }^{2}$ Matched peptides supporting the protein identification. ${ }^{3}$ Retrieved theoretical value. ${ }^{4}$ Species from which the matching toxins were identified.

\section{Authors' contributions}

EBN, GRC, JMS and DCP conceived this research and designed experiments, participated in the design and interpretation of the data, performed experiments and analysis, wrote the paper and participated in the revisions. All authors read and approved the final manuscript.

\section{Ethics approval}

Not applicable.

\section{Consent for publication}

Not applicable.

\section{Supplementary material}

The following online material is available for this article:

Additional file 1. Other proteins matched to the proteomically identified toxins from $N$. mandalayensis venom.

\section{References}

1. Slowinski JB, Wüster W. A new cobra (Elapidae: Naja) from Myanmar (Burma). Herpetologica. 2000;56(2):257-70.

2. Pe T, Htut T, Myint AA, Tun MT. Venom ophthalmia following spitting of venom into the eyes by spitting cobra (Naja mandalayensis) in Myanmar. Myanmar Health Sci Res J. 2002;14(1-3):42-4.

3. Sai-Sein-Lin-Oo, Myat-Thet-Nwe, Khin-Maung-Gyi, Than-Aye, Mi-MiKhine, Myat-Myat-Thein, Myo-Thant, Pyae-Phyo-Aung, Oakkar-KyawKhant, Aye-Zarchi-San, Du-Wun-Moe, Htay-Aung, O'Shea M, Mahmood MA, Peh CA, White J, Warrell DA. Clinical importance of the Mandalay spitting cobra (Naja mandalayensis) in Upper Myanmar - bites, envenoming and ophthalmia. Toxicon. 2020 Sep;184:39-47.

4. Chu ER, Weinstein SA, White J, Warrell DA. Venom ophthalmia caused by venoms of spitting elapid and other snakes: report of ten cases with review of epidemiology, clinical features, pathophysiology and management. Toxicon. 2010 Sep 1;56(3):259-72.

5. Schioldann E, Mahmood MA, Kyaw MM, Halliday D, Thwin KT, Chit NN, Cumming R, Bacon D, Alfred S, White J, Warrell D, Peh CA. Why snakebite patients in Myanmar seek traditional healers despite availability of biomedical care at hospitals? Community perspectives on reasons. PLoS Negl Trop Dis. 2018 Feb 28;12(2):e0006299.

6. Fusco LS, Neto EB, Francisco AF, Alfonso J, Soares A, Pimenta DC, Leiva LC. Fast venomic analysis of Crotalus durissus terrificus from northeastern Argentina. Toxicon X. 2020 Jun 15;7:100047. doi:10.1016/j. toxcx.2020.100047.

7. Mariano DOC, Prezotto-Neto JP, Spencer PJ, Sciani JM, Pimenta DC. Proteomic analysis of soluble proteins retrieved from Duttaphrynus melanostictus skin secretion by IEx-batch sample preparation.J Proteomics. 2019 Oct 30;209:103525. doi: 10.1016/j.jprot.2019.103525. Epub 2019 Sep 14.
8. de Oliveira LA, Ferreira RS Jr, Barraviera B, de Carvalho FCT, de Barros LC, Dos Santos LD, Pimenta DC. Crotalus durissus terrificus crotapotin naturally displays preferred positions for amino acid substitutions. J Venom Anim Toxins incl Trop Dis. 2017 Nov 28;23:46. doi:10.1186/s40409-017-0136-5.

9. Ferreira RS Jr, Sciani JM, Marques-Porto R, Junior AL, Orsi R O, Barraviera $B$, Pimenta DC. Africanized honey bee (Apis mellifera) venom profiling: seasonal variation of melittin and phospholipase A(2) levels. Toxicon. 2010 Sep 1;56(3):355-362. doi: 10.1016/j.toxicon.2010.03.023. Epub 2010 Apr 18.

10. Kazandjian TD, Petras D, Robinson SD, van Thiel J, Greene HW, Arbuckle K, Barlow A, Carter DA, Wouters RM, Whiteley G, Wagstaff SC, Arias AS, Albulescu LO, Plettenberg Laing A, Hall C, Heap A, Penrhyn-Lowe $S$, McCabe CV, Ainsworth S, da Silva RR, Dorrestein PC, Richardson MK, Gutiérrez JM, Calvete JJ, Harrison RA, Vetter I, Undheim EAB, Wüster W, Casewell NR. Convergent evolution of pain-inducing defensive venom components in spitting cobras. Science. 2021 Jan 22;371(6527):386-390. doi: 10.1126/science.abb9303.

11. Vejayan J, Khoon TL, Ibrahim H. Comparative analysis of the venom proteome of four important Malaysian snake species. J Venom Anim Toxins incl Trop Dis. 2014 mar 4;20(1):6.

12. Hus KK, Buczkowicz J, Petrilla V, Petrillová M, Łyskowski A, Legáth J, Bocian A. First look at the venom of Naja ashei. Molecules. 2018 Mar 8;23(3):609.

13. Sánchez A, Herrera M, Villalta M, Solano D, Segura Á, Lomonte B, Gutiérrez JM, León G, Vargas M. Proteomic and toxinological characterization of the venom of the South African Ringhals cobra Hemachatus haemachatus. J Proteomics. 2018 Jun 15;181:104-17.

14. Hamako J, Matsui T, Nishida S, Nomura S, Fujimura Y, Ito M, Ozeki Y, Titani K. Purification and characterization of kaouthiagin, a von Willebrand factor-binding and -cleaving metalloproteinase from Naja kaouthia cobra venom. Thromb Haemost. 1998 Sep;80(3):499-505.

15. Ward CM, Andrews RK, Smith Al, Berndt MC. Mocarhagin, a novel cobra venom metalloproteinase, cleaves the platelet von Willebrand factor receptor glycoprotein Ibalpha. Identification of the sulfated tyrosine/ anionic sequence Tyr-276-Glu-282 of glycoprotein Ibalpha as a binding site for von Willebrand factor and alpha-thrombin. Biochemistry. 1996 Apr 16;35(15):4929-38. doi: 10.1021/bi952456c.

16. Sun QY, Wang CE, Li YN, Bao J. Inhibition of platelet aggregation and blood coagulation by a P-III class metalloproteinase purified from Naja atra venom. Toxicon. 2020 Sep 21;187:223-31.

17. De Luca M, Dunlop LC, Andrews RK, Flannery JV Jr, Ettling R, Cumming DA, Veldman GM, Berndt MC. A novel cobra venom metalloproteinase, mocarhagin, cleaves a 10 -amino acid peptide from the mature $\mathrm{N}$ terminus of P-selectin glycoprotein ligand receptor, PSGL-1, and abolishes P-selectin binding. J Biol Chem. 1995 Nov 10;270(45):26734-7. doi: 10.1074/ jbc.270.45.26734. PMID: 7592904.

18. Ward CM, Vinogradov DV, Andrews RK, Berndt MC. Characterization of mocarhagin, a cobra venom metalloproteinase from Naja mocambique mocambique, and related proteins from other Elapidae venoms. Toxicon. 1996 Oct;34(10):1203-6. doi: 10.1016/0041-0101(96)00115-8. PMID: 8931262.

19. Jarvis GE, Atkinson BT, Frampton J, Watson SP. Thrombin-induced conversion of fibrinogen to fibrin results in rapid platelet trapping which is not dependent on platelet activation or GPlb. Br J Pharmacol. 2003 Feb;138(4):574-83. doi: 10.1038/sj.bjp.0705095. PMID: 12598411; PMCID: PMC1573703.

20. Li R, Zhu S, Wu J, Wang W, Lu Q, Clemetson KJ. L-amino acid oxidase from Naja atra venom activates and binds to human platelets. Acta Biochim Biophys Sin (Shanghai). 2008 Jan;40(1):19-26. 
21. Chwetzoff S, Tsunasawa S, Sakiyama F, Menez A. Nigexine, a phospholipase A2 from cobra venom with cytotoxic properties not related to esterase activity. Purification, amino acid sequence, and biological properties. J Biol Chem. 1989 Aug 5;264(22):13289-97.

22. Rowan EG, Harvey AL, Menez A. Neuromuscular effects of nigexine, a basic phospholipase A2 from Naja nigricollis venom. Toxicon. 1991;29(3):371-4.

23. Bhat MK, Gowda TV. Isolation and characterization of a lethal phospholipase A2 (NN-IVb1-PLA2) from the Indian cobra (Naja naja naja) venom. Biochem Int. 1991 Dec;25(6):1023-34.

24. Halim HY, Shaban EA, Hagag MM, Daoud EW, el-Asmar MF. Purification and characterization of phosphodiesterase (exonuclease) from Cerastes cerastes (Egyptian sand viper) venom. Toxicon. 1987;25(11):1199-207. doi: 10.1016/0041-0101(87)90138-3.

25. Tan $\mathrm{CH}$, Tan KY, Fung SY, Tan NH. Venom-gland transcriptome and venom proteome of the Malaysian king cobra (Ophiophagus hannah). BMC Genomics. 2015 Sep 10;16(1):687.

26. Lin CC, Wu BS, Wu WG. Crystal structure of snake venom phosphodiesterase (PDE) from Taiwan cobra (Naja atra atra) in complex with AMP. 26 Sep 2016 [cited 25 May 2021]. In: Worldwide Protein Databank (wwPDB) [Internet]. Piscataway (NJ): wwPDB, 2017. DOI: 10.2210/pdb5gz5/pdb. Record n.: 5GZ5.

27. Hart ML, Köhler D, Eckle T, Kloor D, Stahl GL, Eltzschig HK. Direct treatment of mouse or human blood with soluble 5'otidase inhibits platelet aggregation. Arterioscler Thromb Vasc Biol. 2008 Aug;28(8):1477-83.

28. Dhananjaya BL, D'Souza CJM. The pharmacological role of nucleotidases in snake venoms. Cell Biochem Funct. 2010 Apr;28(3):171-7.

29. Trummal K, Samel M, Aaspõllu A, Tõnismägi K, Titma T, Subbi J, Siigur J, Siigur E. 5'-Nucleotidase from Vipera lebetina venom. Toxicon. 2015 Jan;93:155-63. doi: 10.1016/j.toxicon.2014.11.234. Epub 2014 Nov 28.

30. Mitra J, Bhattacharyya D. Phosphodiesterase from Daboia russelli russelli venom: purification, partial characterization and inhibition of platelet aggregation. Toxicon. 2014 Sep;88:1-10. doi: 10.1016/j.toxicon.2014.06.004. Epub 2014 Jun 14
31. Diviani D, Osman H, Delaunay M, Kaiser S. The role of A-kinase anchoring proteins in cardiac oxidative stress. Biochem. Soc Trans. 2019 Oct 31;47(5):1341-53.

32. Chanda A, Patra A, Kalita B, Mukherjee AK. Proteomics analysis to compare the venom composition between Naja naja and Naja kaouthia from the same geographical location of eastern India: correlation with pathophysiology of envenomation and immunological cross-reactivity towards commercial polyantivenom. Expert Rev Proteomics. 2018 Nov;15(11):949-961.

33. Gasanov SE, Shrivastava IH, Israilov FS, Kim AA, Rylova KA, Zhang B, Dagda RK. Naja naja oxiana cobra venom cytotoxins CTI and CTII disrupt mitochondrial membrane integrity: implications for basic three-fingered cytotoxins. PLoS One. 2015 Jun 19;10(6):e0129248.

34. Dufton MJ, Hider RC. Structure and pharmacology of elapid cytotoxins. Pharmacol Ther. 1988;36(1):1-40.

35. Suzuki-Matsubara M, Athauda SBP, Suzuki Y, Matsubara K, Moriyama A. Comparison of the primary structures, cytotoxicities, and affinities to phospholipids of five kinds of cytotoxins from the venom of Indian cobra, Naja naja. Comp Biochem Physiol C Toxicol Pharmacol. 2016 Jan;179:158-64.

36. Harvey AL, Bradley KN, Cochran SA, Rowan EG, Pratt JA, Quillfeldt JA, Jerusalinsky. What can toxins tell us for drug discovery? Toxicon. 1998 Nov;36(11):1635-40.

37. Tsetlin V. Snake venom a-neurotoxins and other "three-finger" proteins. Eur J Biochem. 1999 Sep;264(2):281-6.

38. Osipov AV, Levashov MY, Tsetlin VI, Utkin YN. Cobra venom contains a pool of cysteine-rich secretory proteins. Biochem Biophys Res Commun. 2005 Mar 4;328(1):177-82.

39. Brillard-Bourdet M, Nguyên V, Ferrer-di Martino M, Gauthier F, Moreau T. Purification and characterization of a new cystatin inhibitor from Taiwan cobra (Naja naja atra) venom. Biochem J. 1998 Apr 1;331(Pt 1):239-44. doi: 10.1042/bj3310239.

40. Ritonja A, Turk V, Gubenšek F. Serine proteinase inhibitors from Vipera ammodytes venom: isolation and kinetic studies. Eur J Biochem. 1983 Jun 15;133(2):427-32. 